\title{
Dimensionless Regime Mapping and Modeling for Particle Suspension Flows
}

\author{
Li Xiao ${ }^{1}$, Haolin Zhang ${ }^{2}$, Xi Wang ${ }^{3}$, Feifei Zhang ${ }^{4}$, \\ ${ }^{1,2}$ Sinopec Research Institute of Petroleum Engineering \\ ${ }^{3,4}$ Yangtze University
}

*Corresponding Author: Feifei Zhang, Yangtze University

\begin{abstract}
In this paper, the particle suspension flows in pipes are classified into three major regimes of: flow with a stationary particle bed, flow with moving particle bed or dunes, and flow of fully dispersed particle-liquid mixture. It is shown that these regimes can be characterized by only two dimensionless parameters, which are the ratios of the characteristic flow velocity and particle shear velocity, and projection of the particle settling velocity on the direction of the flow. The dimensionless flow regime maps for both circular pipe flow and annulus flow are built using available experimental data and applicable to a wide range of flow parameters. In the considered experiments, the size of the solid particles varied from several micrometers to $1 \mathrm{~cm}$, the particles fraction varied from less than $1 \%$ to approximately $40 \%$, and the solid phase was formed by different particles, such as glass spheres (1.24 sg) and sand (2.5 to $3.0 \mathrm{sg})$. The inclination angle of the flow changed from 0 to $180^{\circ}$. A methodology using suggested mapping for pipe and annulus flow calculations is described in detail.
\end{abstract}

Keywords: dimensionless mapping, solid suspension, particle transport, flow regime, annulus flow, pipe flow

\section{INTRODUCTION}

Transport of particle suspensions in liquids can be encountered in a variety of industrial processes, including many operations in mining, civil, and oil and gas industries. The types of particle distribution in the flow, which are called the particle flow patterns, depend on the suspension flow parameters. Flow patterns are critically important for successful operations. For example, high particle volume fraction or significant particle deposition in the flow could cause severe pressure loss and block the flow conduit, often resulting in damaging the system and even accidents. Accurate predictions and monitoring of the flow patterns are extremely important during the design and operation in real life applications.

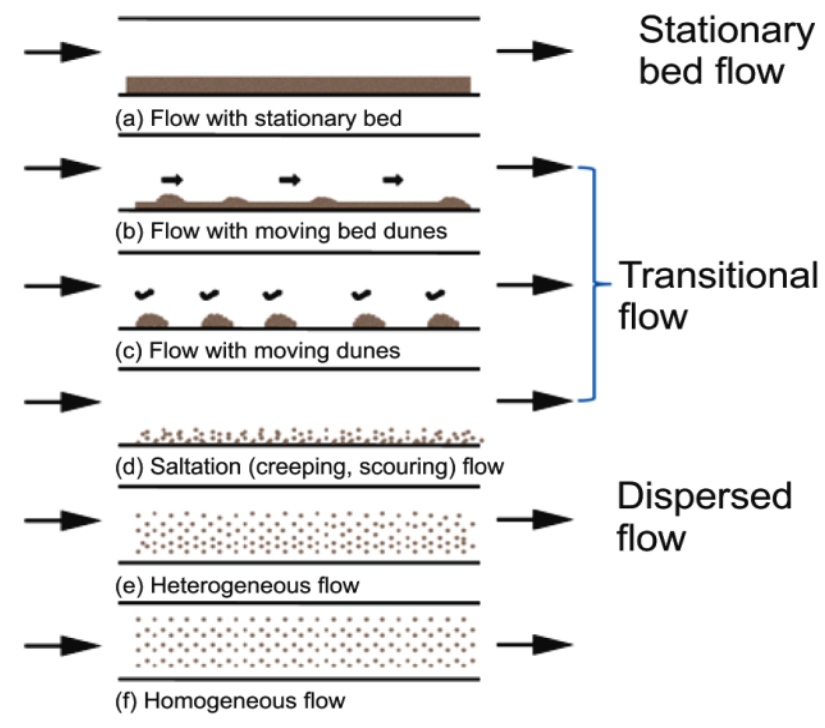

Figure1. Schematic configuration of the flow patterns [7] 
There are several different parameters suggested for describing various flow patterns of solid-liquid mixtures [1-6]. In general, these flow patterns can be divided into four categories: flows with stationary particle beds, flows with moving bed or dunes, flows with moving dunes, and the saltation, or creeping flow, as shown in Figure 1 [7]. The second and third flow patterns can be considered as a transition from stationary bed flow to dispersed flow. In this study, these two flow patterns are combined and named as transitional flow. The stationary bed flow has a stationary packed particle bed at the bottom of the flow conduit with flow of particle liquid mixture above. In the dispersed flow, all particles are fully dispersed in the flow and there are no packed particle beds in the conduit. The particle distribution can be uniform or non-uniform. The transitional flow is the transition between stationary bed flow and dispersed flow, with moving particle beds and dunes.

\subsection{Previous Flow Pattern Maps}

The flow patterns depend on various parameters, such as solid particle shape and size, solid density, solid fraction in the flow, flow conduit shape and size, flow conduit inclination angle, fluid velocity, and fluid viscosity and density. Traditional approaches to predict the flow patterns are conducting a series of experimental tests and plot the flow patterns using superficial particle phase velocity and carrier fluid velocity, as shown in Figure2 [4]. These flow pattern maps are only valid within their test range. In various applications, the size of the particles varies from tens of microns to several centimeters. The fluid properties (viscosity, density, Newtonian, non-Newtonian) and the flow conduit properties (shape, size, and inclination angle) also change significantly for different operations. It is impossible to conduct experimental tests to cover all the parameter ranges.

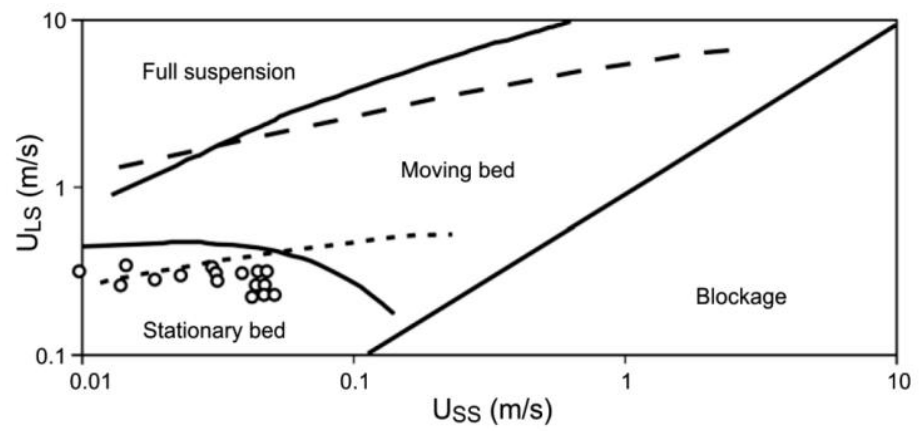

Figure2. Example of the flow pattern map plotted by superficial velocities [4].

Another approach involves plotting the flow pattern map using the pressure gradient of the flow and the mixture velocity, as shown in Figure 3 [7]. At low flow velocity conditions, the particles tend to pack in the conduit and form a stationary or moving particle bed. The formation of the bed or dunes decreases the flow area and leads to high pressure loss. As the flow velocity increases, the pressure gradient decreases initially, and then increases after it reaches a minimum point, as shown in Figure 3. Below the minimum point, the pressure gradient is dominated by particles; beyond the minimum point, the pressure gradient is dominated by friction loss of the flow. This flow pattern plotting approach has broader application than approach of the previous work [4], but is still restricted by the experimental range because the two axes are dimensional.

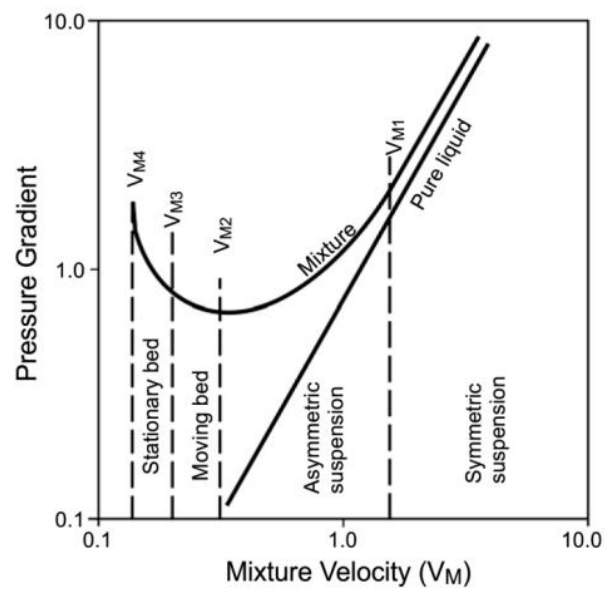

Figure3. Schematic representation of flow patterns and pressure gradient [7]. 


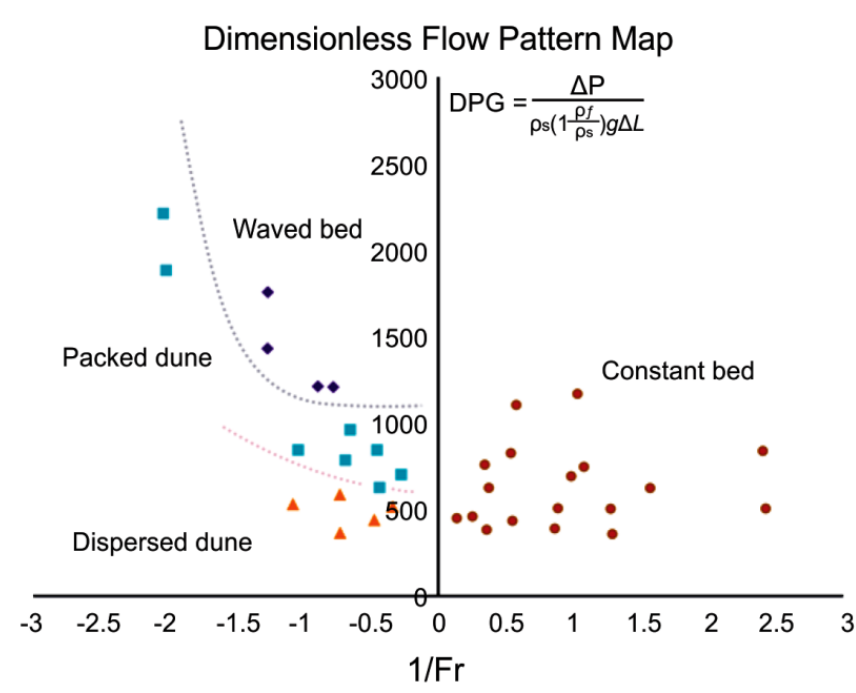

Figure4. Dimensionless flow regime map [6]

Recently, Zhang (2015) introduced a dimensionless flow regime map, as shown in Figure 4. The two parameters used are dimensionless pressure gradient and a modified Froude number (Fr). This flow pattern map is more general, because the map axes are dimensionless. It is also valid for inclined conduits because the modified Froude number includes the conduit inclination angle [6].

This paper suggests two other dimensionless parameters based on analysis of the equations for particle motion in the fluid flow. Using these two dimensionless parameters, a generalized flow pattern mapping method is developed to analyze the two phase suspension flow patterns.

\section{Particle Motion In Fluid Flow}

The analysis of single particle movement in fluid flow is necessary for understanding of the major factors affecting suspension flow. For a single particle in a dilute suspension, there are three different phenomena influencing its motion represented by three different velocities: as shown in Figure 5, the particle settling under the action of the gravity force, the particle mixing and resuspension due to the action of turbulence, and particle translation in axial direction of the pipe under the action of viscous drag.

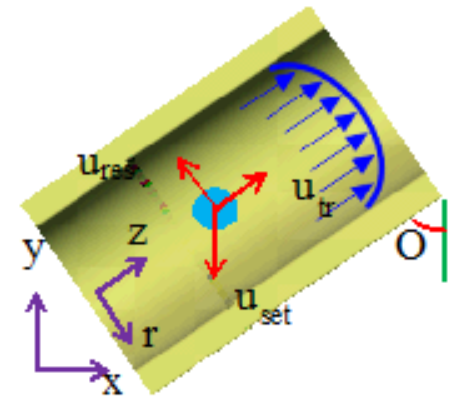

Figure5. Single particle moving in suspension circular pipe flow

The settling velocity $\mathrm{u}_{\mathrm{set}}$ can be obtained from the balance between the particle weight, the Archemedes force, and the drag force in vertical direction caused by particle settling, which is represented by Eq. $1[8]$.

$u_{s e t}=-\sqrt{\frac{8 G_{e}}{\rho_{f} C_{D}^{\prime} \pi d^{2}}}=-\sqrt{\frac{32\left(\rho_{p}-\rho_{f}\right) g d_{p}}{3 \rho_{f} C_{D}^{\prime}}}$

where $\square_{p}$ an $\square_{f}$ are the particle and fluid density, respectfully, $d_{p}$ is the particle diameter, and $C_{D}^{\prime}$ is the drag coefficient of the particle at velocity $\mathrm{u}_{\text {set }}$. Because the drag coefficient depends on the particle Reynolds number, which depends on the particle settling velocity, Eq. 1 is needs to be solved in respect to $\mathrm{u}_{\mathrm{set}}$, for example, by iterations.

The particle resuspension is caused by the lift forces caused by the following effects: (1) unequal pressure distribution and the shear stress around individual particle surface, (2) the turbulent eddies, 
(3) and the particle-particle interactions [9] as shown in Figure 6. Majority of the suspension flows in the industrial applications are turbulent.

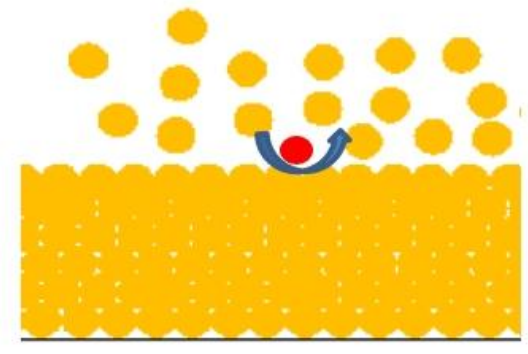

Figure6. Re-suspension at the surface of the packed particles

The average mixture density $\rho_{\mathrm{m}}$ is defined by Eq. 3, and the shear stress $\tau_{\mathrm{m}}$ can be obtained by using Eq. 4.

$$
\begin{aligned}
& \rho_{m}=\rho_{f}\left(1-C_{c}\right)+\rho_{p} C_{c} \\
& \tau_{m}=\frac{\rho_{m} f_{m} u_{m}^{2}}{2}
\end{aligned}
$$

where $\mathrm{C}_{\mathrm{c}}$ is the local particle concentration, $f_{m}$ is the friction factor between mixture flow and packed particles, and $u_{m}$ is the mixture velocity. The correlations provided by Televantos [12] (1979) and Ozbayoglu [13] (2002) can be used to cover the effect of wall roughness and presence of particles in the flow. Televantos correlation can be represented by Eq. 5, which is developed for pipe flow condition.

$$
\frac{1}{\sqrt{2 f_{m}}}=-0.86 \ln \left(\frac{\frac{d_{p}}{d_{h y d}}}{3.7}+\frac{2.51}{\operatorname{Re} \sqrt{2 f_{m}}}\right)
$$

where $d_{p}$ is the particle diameter, $d_{\text {hyd }}$ is the hydraulic diameter of the flow conduit, and Re is the Reynolds number of the flow. Ozbayoglu modified Televantos correlation for annulus flow by introducing a characteristic diameter, which can be represented by Eq. 6 .

$f_{m}=f_{m}\left(\frac{d_{p} \sqrt[3]{\frac{C_{c}}{C_{p a c}}}}{2 d_{p}}+1\right)$

where $\mathrm{C}_{\mathrm{c}}$ is the suspended local particle concentration, and $\mathrm{C}_{\mathrm{pac}}$ is the packed particle concentration.

In the cases of no packed particles in the flow (the dispersed flow regime), the shear stress at the pipe wall can be used to calculate the shear velocity $u^{*}$.

Thus, the entrainment process can be characterized by the ratio

$$
\Omega_{h}=\frac{u^{*}}{u_{\text {set }} \sin \theta}
$$

The denominator in Eq. 7 equals to projection of the particle settling velocity on the normal to the flow direction, accounting for the pipe inclination. For example, one of the first criteria of particle entrainment, proposed by Bagnold (1966) for horizontal pipes ( $\sin \square=1$ ), can be written simply as

$$
\Omega_{h}=1
$$

The parameter $\square_{h}$ is a characteristic ratio of the turbulent lift force and forces that oppose particle motion, such as gravitational and viscous forces acting on the particle.

By analogy, the impact of particle sedimentation on particle transport in pipes can be characterized by the ratio

$$
\Omega_{v}=\frac{u_{f}}{u_{s e t} \sin \theta}
$$




\section{Generalized Dimensionless Flow Regime MAP}

As mentioned in the previous section, all the flow patterns are classified into three different flow regimes: flow with a stationary particle bed, flow with moving particle bed or dunes, and flow with completely dispersed particles. In this section, two flow regime maps are introduced by plotting the available experimental data using the two dimensionless parameters, $\Omega_{v}$ and $\Omega_{h}$, discussed in the previous section.

In Figure 7, the available experimental data for pipe flow from several different studies [8,14-17] are plotted against two dimensionless parameters, $\square_{h}$ and $\square_{v}$. The data used to build these maps is taken from studies with significantly different experimental conditions. For example, the size of the particles varied from several micrometers to one centimeter, while the particle volume fraction varied from less than 1 to approximately $40 \%$, and the particles were glass spheres $(1.24 \mathrm{sg})$ and sand particles $(2.5 \sim 3.0 \mathrm{sg})$. The inclination angle of the flow conduits changed from 0 to $180^{\circ}\left(0 \sim 90^{\circ}\right.$ for upward flow, and $90 \sim 180^{\circ}$ for downward flow).

Figure 8 shows the flow regime map for annulus flow. Unfortunately, available data is for annulus flow is scarcer than that for the pipe flow because there have been far fewer studies with particle suspension flows in annuli. The data in this figure is from Zhang's (2015) study for annulus flow. The average size of the solid particles is $3 \mathrm{~mm}$, particle volume concentration varies from 5 to approximately $40 \%$, and the fluid used is water. Inclination angle of the flow conduits changes from 30 to $90^{\circ}$.

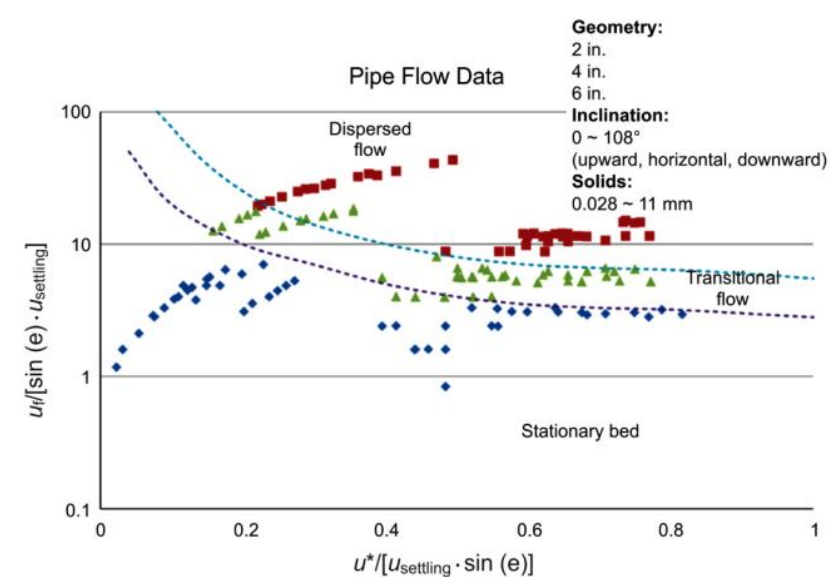

Figure7. Proposed dimensional flow regime map for pipe flow

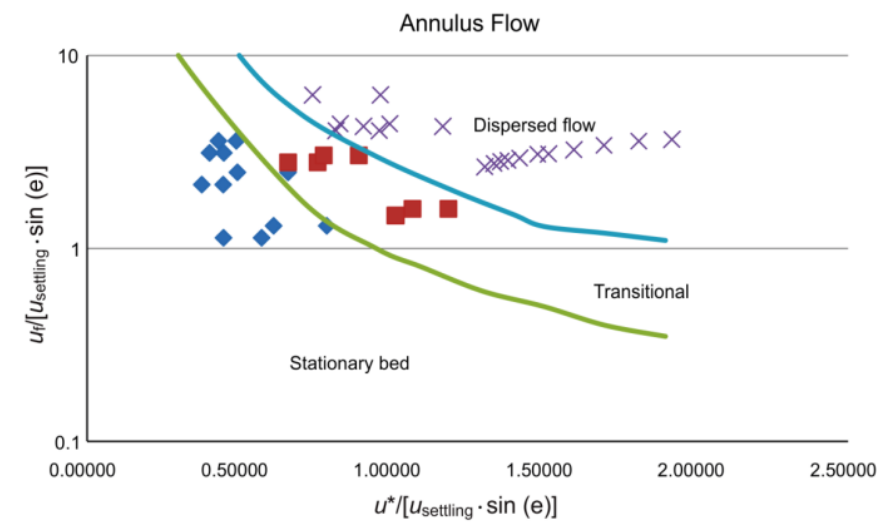

Figure8. Proposed dimensional flow regime map for annulus flow

The two dimensionless parameters characterizing solid phase transport and are used to plot a generalized flow regime map. This way, the flow regime map is valid for different particle sizes and materials, different conduits, and different fluids, which significantly reduces the number of experimental tests required to predict flow regime in practical applications.

The vertical axis represents the balance between the particle translational flow and sedimentation; the horizontal axis represents the balance between the particle sedimentation and entrainment by the turbulent eddies. Thus, these two dimensionless parameters are a combination of the major parameters that affect the two phase flow regime. While particle properties (size, shape, and density) are included 
in the settling velocity, $\mathrm{u}_{\mathrm{set}}$, the fluid properties (density and viscosity) are included in the settling velocity and the friction velocity, $\mathrm{u}^{*}$. Particle concentration and conduit geometry are also accounted for by the friction velocity and by the term $\sin \theta$. For vertical flow $(\theta=0), \sin \theta$ equals to zero, so both $\Omega_{v}$ and $\Omega_{h}$ are infinite, which indicates the dispersed flow regime according to maps shown in Figures 7 and 8 . It is remarkable that all available experimental data on particle suspension flows can be plotted using just two dimensionless parameters, with clear boundaries between different flow regimes. This fact significantly simplifies the suspension flow simulations using advanced mechanistic models.

\section{MECHANISTIC MODELS}

The shear velocity in the dimensionless number $\Omega_{h}$ cannot be directly measured, but can be obtained by the mechanistic modeling for given flow conditions. The mechanistic models account for phase mass balance and momentum balance.

\subsection{Stationary Bed Flow}

For stationary bed flow scenario, layer models $[4,18]$ can be used to calculate the shear velocity between slurry flow and packed particles. One of the most important assumptions in the layer models is that the thickness of each layer is constant and interfaces between different layers are smooth. The model is based on the mass and momentum conservation of each section (packed particles, particlefluid mixture, and fluid) in the flow regime. Figure 9 shows a schematic of the geometry for the most complex regime, which has three sections: pure fluid layer, mixture layer, and particle bed layer. The formulations of the mechanistic model are shown in the following.

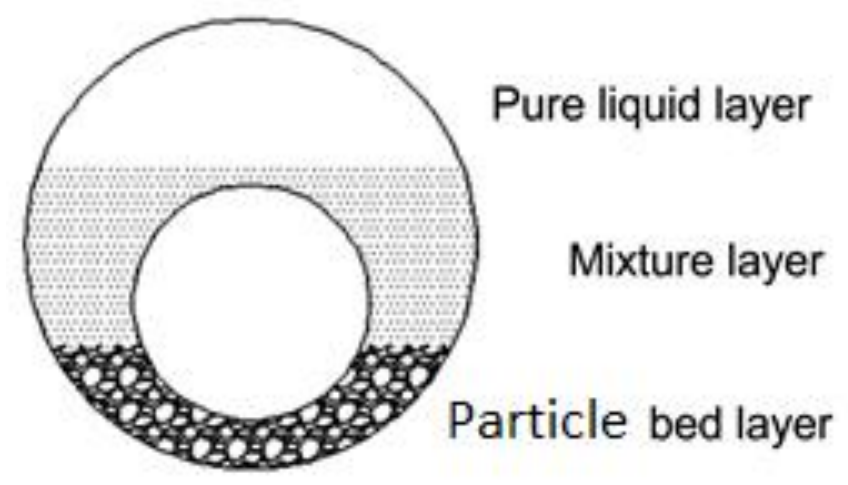

Figure9. Layer model geometry

The mass conservation for the particle phase is

$\rho_{s} A_{s b} \varnothing U_{s b}+\rho_{s} A_{m} C_{m} U_{m s}=\rho_{s} A U_{s s}$.

The mass conservation for fluid phase is

$\rho_{f} A_{u} U_{u}+\rho_{L} A_{m}\left(1-C_{m}\right) U_{m f}+\rho_{L} A_{s b}(1-\emptyset)\left(U_{s b}+\mathrm{U}_{\mathrm{r}}\right)=\rho_{L} Q$

(11) The momentum conservations for the three sections in the geometry are

Upper fluid layer: $-A_{u}\left(\frac{\Delta P}{L}\right)=-\tau_{u} S_{u}-\tau_{u_{-}-m} S_{u_{-} m}-\rho_{u} g A_{u} \cos \theta$

Mixture layer: $-A_{m}\left(\frac{\Delta P}{L}\right)=-\tau_{m} S_{m}+\tau_{u_{\_}} S_{u_{-} m}-\tau_{m_{\_} s b} S_{m_{-} s b}-\rho_{m} g A_{m} \cos \theta$

Particle bed layer: $-\mathrm{A}_{\mathrm{sb}}\left(\frac{\Delta \mathrm{P}}{\mathrm{L}}\right)=-\tau_{\mathrm{sb}} \mathrm{S}_{\mathrm{sb}}+\tau_{\mathrm{m}_{-} \mathrm{sb}} \mathrm{S}_{\mathrm{m}_{-} \mathrm{sb}}-\rho_{\mathrm{sb}} \mathrm{g} \mathrm{A}_{\mathrm{sb}} \cos \theta$

where $\mathrm{A}$ is the total cross-sectional area, $\frac{\Delta \mathrm{P}}{\mathrm{L}}$ is the pressure gradient, $\tau$ is the shear stress, $\mathrm{S}$ is the perimeter of each section, $\rho$ is the density, and $\theta$ is the conduit inclination angle. The subscript $u$ represents the upper pure fluid layer, $\mathrm{m}$ represents the mixture layer, sb represents the solid bed layer, $\mathrm{u} \_\mathrm{m}$ is the interface between upper layer and mixture layer, and $\mathrm{m} \_s b$ represents the interface between the mixture layer and solid bed layer.

For stationary bed flow regime, the velocity of the particle bed layer is zero, so it can be eliminated from the calculation. If the pure liquid layer does not exist, the model can be simplified to two-layer (a slurry layer and a solid bed layer). Detailed discussions and applications of this model can be found in previous studies $[4,13]$. In this particular model, the shear stress between the mixture layer and the particle bed layer $\tau_{\mathrm{m}_{-} \mathrm{sb}}$ should be used to calculate the shear velocity in the dimensionless number $\Omega_{h}$. 


\subsection{Transitional Flow}

For the transitional flow regime scenario, the choice of mechanistic models depends on the smoothness of the particle bed. For horizontal or near horizontal flow $\left(55^{\circ} \leq \theta \leq 125^{\circ}\right)$, the surfaces of the packed particle beds or dunes are smooth, and can be modeled by using the layer models discussed in the previous section. The difference with the stationary bed is that the particle bed is moving. For intermediate inclined or near vertical flow $\left(0^{\circ}<\theta<55^{\circ}\right.$ or $\left.125^{\circ}<\theta<180^{\circ}\right)$, the packed particles tend to form discontinuous dunes instead of particles bed. The constant thickness assumption in the layer models is no longer valid in this case. A segment model proposed by Zhang et al [19] can be used in the flow simulation for the dune scenario. A description of the model is shown in Figure 10.

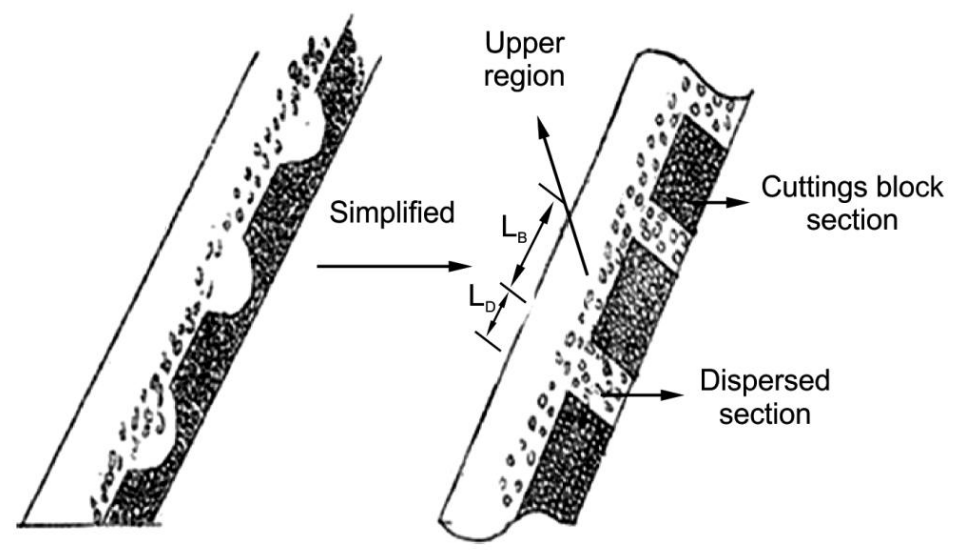

Figure10. Sketch of the segment model [6]

This model includes three segments: an upper section, a dispersed section, and particle dune (block) section. In the upper section, particles are fully dispersed and the flow is comprised by the particles and fluid slurry. The dispersed section is a unique region for inclined flow scenario, which has particles sliding in the counter flow direction. The particle block section is comprised by packed particles, and has a shape of dunes. This segment model is based on the conservations of mass for each phase, conservations of momentum for each section, and some the closure relationships. A detailed description of the model can be found in the original paper [6], which would not be repeated here.

\subsection{Dispersed Flow}

For the dispersed flow regime, the wall shear stress can be used to obtain the shear velocity in the dimensionless number $\Omega_{h}$ because there are no packed particles in this flow regime. The models and correlations for slurry flow calculation $[8,20,21]$ can be used to obtain the pressure gradient of the flow, which can be used to calculate the average shear stress at the wall depending on the flow conditions. Then, the shear velocity can be obtained using Eq. 15.

$u^{*}=\sqrt{\frac{\Delta p}{\Delta l S \rho_{m}}}$

where $\Delta p / \Delta l$ is the pressure gradient, $\mathrm{S}$ is the perimeter of the flow conduit, and $\rho_{m}$ is the mixture density of the flow.

\section{Flow Regime Prediction Process}

Because both the flow regime and the pressure loss are initially unknown, an iteration process can be used for predicting the flow regime. By combining mechanistic models and the flow regime map, the flow regime, pressure gradient, and particles concentration can be found for any given flow parameters. The procedure for the flow regime prediction is the following:

1) Run all three mechanistic models with the given input parameters.

2) Find the flow regime for each model by comparing the outputs from that model with the flow regime map. 
3) Compare the obtained flow regimes with the original flow regime for each mechanistic model. In the case the predicted flow regime is the same as the original flow regime, then the workflow is converged and the corresponding flow regime is the result of simulation.

4) If there is more than one converged flow regime, apply physical boundaries for particle transport to remove the multiple converged values to one converged value.

\subsection{Flow Regime and Pressure Prediction Examples}

The example in Figure 11 shows the flow regime prediction using the proposed workflow in a 2 -in. pipe. It is assumed that the fluid phase is water and the particle phase is sand. Sand density is 2650 $\mathrm{kg} / \mathrm{m}^{3}$, and sand particle diameter is $1 \mathrm{~mm}$. Flow rate changes from 25 to $70 \mathrm{gpm}$ and the inclination angle of the pipe changes from 10 to $100^{\circ}\left(0^{\circ}\right.$ means upward vertical, $90^{\circ}$ means horizontal, and $180^{\circ}$ means downward vertical). In this figure, blue color represents the stationary bed flow regime, green color represents the transitional flow regime, and yellow color represents the dispersed flow regime. This figure shows that particles tend to pack at near horizontal and low flow rate conditions, and tend to disperse at near vertical and high flow rate conditions. When inclination angle is less than $40^{\circ}$, no particle packing appears for all the simulated flow velocities. For horizontal flow (inclination angle is $\left.90^{\circ}\right)$, the particle bed disappears at approximately $2 \mathrm{~m} / \mathrm{s}$.

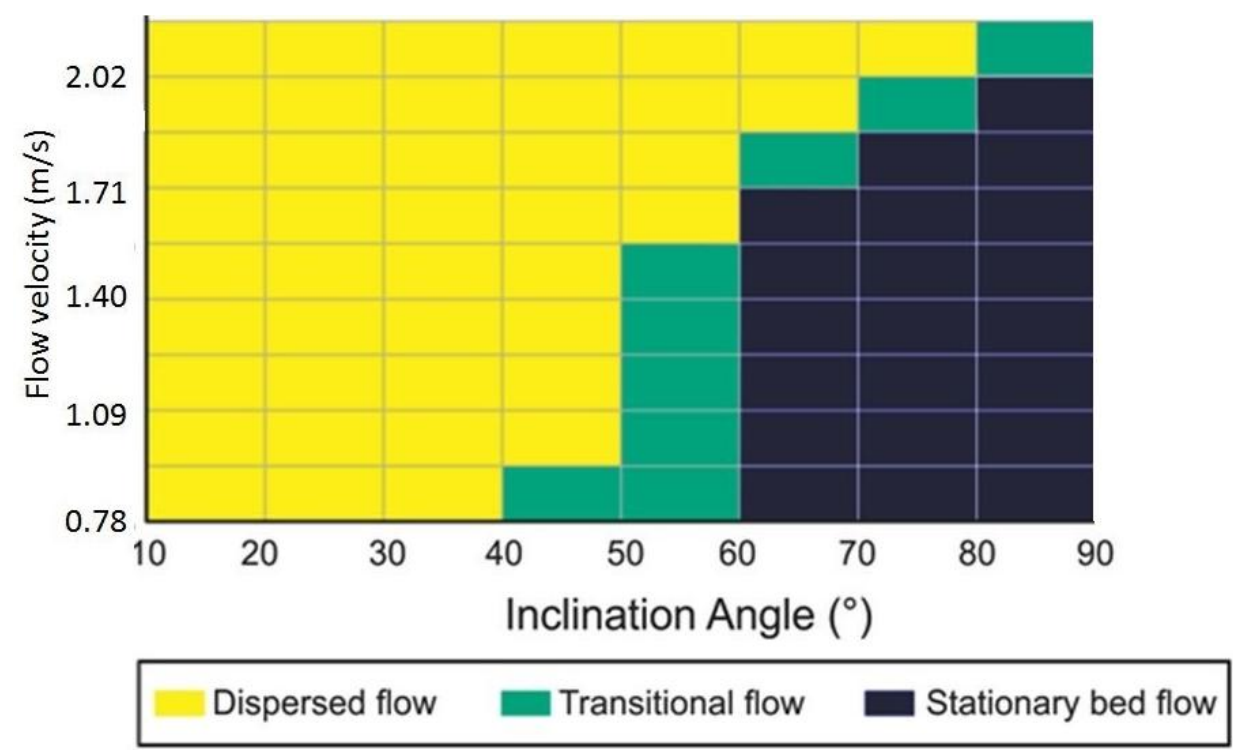

Figure11. Flow regime prediction examples

6-in. Pipe FLow with 0.25-mm Sand

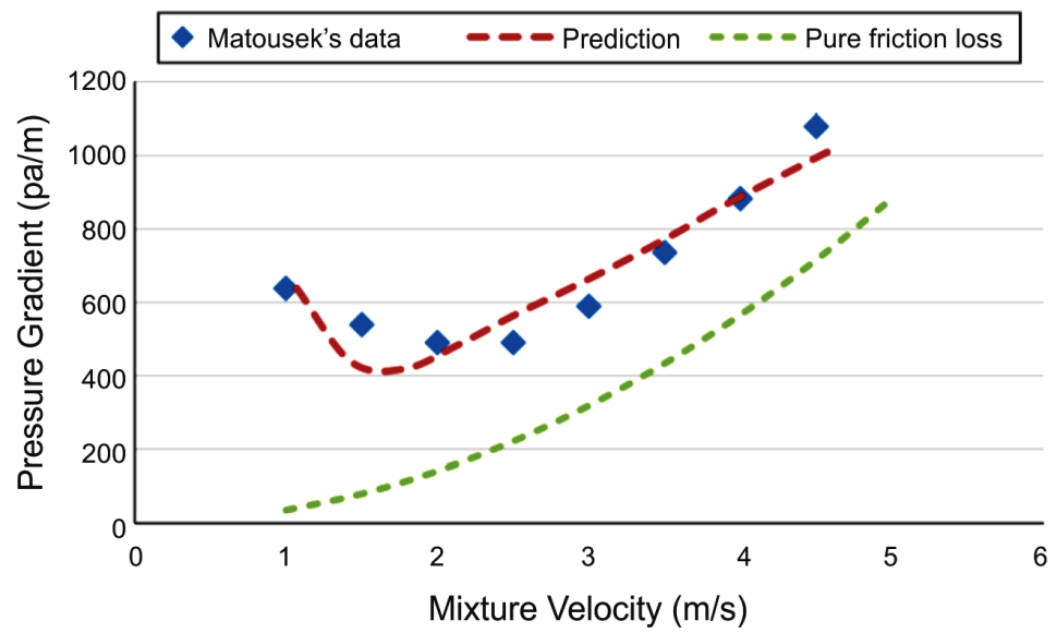

Figure12. Pressure loss validations for horizontal flow

Figure 12 compares the predicted pressure gradients and Matousek's [22] data. The test is conducted in a 6-in. horizontal pipe and the average particle size is $0.25 \mathrm{~mm}$. The fluid used in the test is water. As shown in the figure, the frictional pressure loss for single phase flow increases as flow velocity 
increases. For flow with particles, the pressure loss decreases as flow velocity increases from 1 to 2 $\mathrm{m} / \mathrm{s}$. Beyond $2 \mathrm{~m} / \mathrm{s}$, the pressure loss increases as flow velocity increases. Below $2 \mathrm{~m} / \mathrm{s}$, the flow pattern is the stationary bed regime; beyond $3 \mathrm{~m} / \mathrm{s}$, the flow pattern is the dispersed flow regime. The minimum pressure loss occurs during the transitional flow regime, which is between 2 and $3 \mathrm{~m} / \mathrm{s}$ for this case.

Figure 12 shows the pressure drop of the flow as function of the flow rate at one inclination position. In Figure 13, the predicted pressure drop is plotted against the inclination angle, and compared with the experimental results with Vlasak's (2006) for a fixed flow rate.

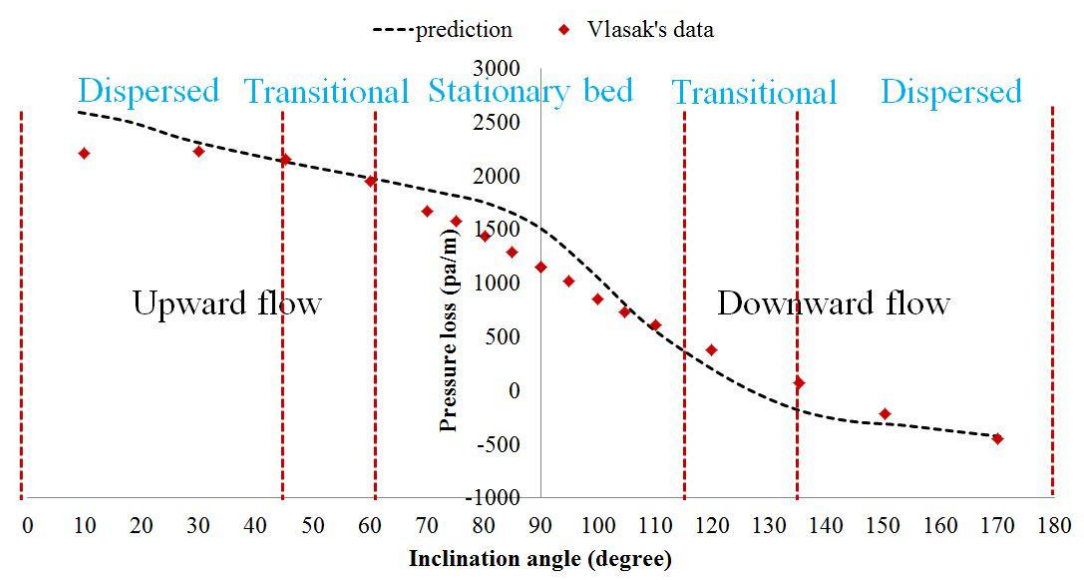

Figure13. Pressure loss validation at different inclined positions

The experimental tests in Figure 13 were conducted in a 4-in pipe using water as the carrier fluid. Particles were $10 \mathrm{~mm}$ sand, which is relative large. Flow velocity was $2.84 \mathrm{~m} / \mathrm{s}$. Inclination range of the tests was 0 to $180^{\circ}$, which is the entire range from upward-vertical flow to downward-vertical flow. The inclination angle $0^{\circ}$ represents upward-vertical flow, $90^{\circ}$ represents the horizontal flow, and $180^{\circ}$ represents downward-vertical flow. The flow is upward between 0 and $90^{\circ}$ and it is downward between 90 and $180^{\circ}$. The pressure loss in Figure 13 is the total pressure loss of the flow subtracted by the static pressure gradient of the pure fluid, which can be represented by Eq. 16 .

$$
-\frac{\Delta P}{\Delta L}=-\frac{\Delta P}{\Delta L_{\text {total }}}+\rho_{f} g \sin \theta
$$

Thus, the pressure drop shown in Figure 13 includes two parts: (1) the frictional loss of the flow, and (2) the change of the average density of the flow caused by the existence of the particles. The average particle concentration in the flow is approximately $8 \%$, which leads to approximately a $13 \%$ increase in flow density. For the upward flow, the total pressure drop is the sum of the absolute value of the frictional pressure drop and pressure drop caused by density change; for the horizontal flow, the total pressure drop is due to the frictional loss, while for the upward flow, the pressure drop is the frictional pressure drop minus the gravitational pressure. These two comparisons illustrate the relationship between the flow pattern and flow pressure drop.

\section{CONCLUSions}

Using analysis of factors affecting particle motion in suspension, it is shown that these regimes can be characterized by only two dimensionless parameters: the ratio between the flow velocity and particle settling velocity, and the ratio between shear velocity and particle settling velocity. The impact of sedimentation on particle transport is characterized by $\Omega_{h}$, which is the ratio between mean flow velocity and particle settling velocity. The particle entrainment process is characterized by $\Omega_{h}$, which is the ratio between shear velocity at the surface of packed particle and the settling velocity. The two dimensionless parameters used to plot the flow regime map represent the three physical factors that control the particle-fluid flow regime: particle settling, particle re-suspension, and particle transport.

The suggested flow regime map can be used for various particle sizes and materials, conduits, and fluids. Major particle properties (size, shape, and density) are included in the settling velocity, $\mathrm{u}_{\text {set }}$, and the fluid properties are (density and viscosity) included in the settling velocity and the friction velocity, $\mathrm{u}^{*}$. The particle concentration and conduit geometry can also affect the friction velocity. The 
conduit inclination angle is also considered by the term $\sin \theta$. For vertical flow $(\theta=0), \sin \theta$ goes to zero, both $\Omega_{v}$ and $\Omega_{h}$ become infinite, which indicates the flow regime is dispersed flow in Figures 7 and 8.

The friction velocity $\mathrm{u}^{*}$ used to plot the flow pattern map can be obtained through mechanistic model. Importantly, the results from flow pattern map enhance the mechanistic model predictions by choosing the right model. The flow regimes, other results, such as particle concentration, particle bed height, and pressure gradient can also be obtained from the workflow.

\section{ACKNOWLEDGEMENT}

The authors would like to thank National Natural Science Foundation of China for the financial support (grant number 51874045) of this project.

\section{REFERENCES}

[1] Vacaldo, J.J. and Charles, M.E. Prediction of pressure gradient for the horizontal turbulent flow of slurries[C]. In Proc. 2th Int. Conf. on the Hydraulic Transport of Solids in Pipes. 1972.

[2] Parzonka, W., Kenchington, J.M., and Charles, M.E. Hydrotransport of solids in horizontal pipes: effects of solids concentration and particle size on the deposit velocity [J]. Can. J. Chem. Engng, 1981, Vol.59: 291-296.

[3] Shamlou, A.P. Hydraulic transport of particulate solids [J]. Chem. Engng Commun, 1987, Vol. 62: 233-249.

[4] Doron, P., Simkhis, M., and Barnea, D. Flow of Solid-Liquid Mixture in Inclined Pipes [J]. Int. J. Multiphase Flow, 1997, Vol. 23(No. 2): 313-323.

[5] Kelessidis, V.C. and Mpandelis, G.E. Flow patterns and minimum suspension velocity for efficient cuttings transport in horizontal and deviated wells in coiled-tubing drilling[C]. SPE/ICoTA Coiled Tubing Conference and Exhibition, 2003.

[6] Zhang, F. Numerical Simulation and Experimental Study of Cuttings Transport in Intermediate Inclined Wells [M]. Ph.D. Dissertation. Tulsa, USA: The University of Tulsa, 2015.

[7] Govier, G.W. and Aziz, K. The Flow of Complex Mixtures in Pipes[C]. Malabar.1972.

[8] Shook, C. A. and Roco, M. C. Slurry Flow: Principles and Practice [M]. Oxford, UK; ButterworthHeinemann, 1991.

[9] Hetsroni, G. Handbook of multiphase systems [M]. Carlsbad, CA: Hemisphere Publishing Corporation, 1982.

[10] Nino, Y., Lopez, F., and Garcia, M. Threshold for Particle Entrainment into Suspensions [J], Sedimentology, 2003, Vol.50: 247-263.

[11] Raudkivi, A.J., Loose Boundary Hydraulics [M]. Pergamon Press, New Nork, $3^{\text {rd }}$ edition, 1990.

[12] Televantos Y., Shook C., Carleton A., Streat M. Flow of Slurries of Coarse Particles at High Solids Concentrations [J], The Canadian Journal of Chemical Engineering, 1979, Vol. 57: 255-262.

[13] Ozbayoglu, M.E. Cuttings Transport with Foam in Horizontal and Highly Inclined Wellbores [M]. PhD dissertation. Tulsa, Oklahoma: The University of Tulsa, 2002.

[14] Admiraal, D. Influence of Pipe Angle on Bed Load Transport in an Inclined Pipe[J]. International Journal of Sediment Research, 2003, Vol. 18(2): 122-129.

[15] Balbino, G. Experimental Investigation of Critical Velocities for Sand Transport in Horizontal Single and Two-phase Flows at Low Sand Concentrations and Comparisons with Existing Models. MS thesis, University of Tulsa, 2010.

[16] Lee, M.S., Matousek, V., Chung, C.K., and Lee, Y.N. Pipe Size Effect on Hydraulic Transport of Jumoonjin Sand -Experiments in a Dredging Test Loop [J]. Journal of Fluid Dynamics, 2013, Vol. 3: 152-162.

[17] Vlasák, P. Chára, Z., Krupička, J., and Konfršt, J. Experimental Investigation of Coarse Particles-Water Mixture Flow in Horizontal and Inclined Pipes [J]. Hydrol. Hydromech., 2006, Vol. 62: 241-247.

[18] Wilson, K.C. Slip Point of Beds in Solids-liquid Pipeline Flow [J]. Proc.ASME. Hyd.Div., 1970, Vol. 96: $1-12$.

[19] Zhang, F., Miska, S., Yu, M., Ozbayoglu, E.M., and Takach, N. Pressure Profile in Annulus: Solids Play a Significant Role [J]. Energy Resour, 2015, Technol Vol. 137(6), 064502

[20] Turian, R.M., and Yuan, T. Flow of Slurries in Pipelines [J]. AIChE Journal, 1977, Vol.23 (No.3): 232-243.

[21] Lareo, C., Fryer, P.J., and Barigou, M. The Fluid Mechanics of Two-Phase Solid-Liquid Food Flows [J]. A Review, Trans IChemE, 1997, Vol.75, Part C.

[22] Matousek, V. Pressure Drops and Flow Patterns in Sand-Mixture Pipes [J]. Experimental Thermal and Fluid Science, 2002, Vol. 26: 693-702. 
[23] Brady, J.F., Khair, A.S., Swaroop, M. On the Bulk Viscosity of Suspensions [J]. Fluid Mech, 2006 , vol. 554: 109-123.

[24] Brennen, C. E. Fundamentals of Multiphase Flow [M]. Cambridge University Press, 2005.

[25] Magarvey, R.H. and Bishop, R.L.Transition Ranges for Three-Dimensional Wakes [J]. Can. J. Phys., 1961, Vol.39: 1418-1422.

[26] Schlichting, H. Boundary Layer Theory [M], McGraw-Hill, New York, 1955.

Citation: Li Xiao, et.al, (2019). "Dimensionless Regime Mapping and Modeling for Particle Suspension Flows", International Journal of Petroleum and Petrochemical Engineering (IJPPE), 5(2), pp.9-19, DOI: http://dx.doi.org/10.20431/2454-7980.0502002

Copyright: (C) 2019 Authors. This is an open-access article distributed under the terms of the Creative Commons Attribution License, which permits unrestricted use, distribution, and reproduction in any medium, provided the original author and source are credited 